\title{
Análise genética de famílias de meios-irmãos de cupuaçuzeiro
}

\author{
Maria Clideana Cabral Maia1, Marcos Deon Vilela de Resende ${ }^{2}$, Luís Cláudio de Oliveira1 ${ }^{1}$, Rafael Moisés Alves ${ }^{3}$, João Luis da \\ Silva Filho ${ }^{4}$, Maurisrael de Moura Rocha ${ }^{5}$, José Jaime Vasconcelos Cavalcante ${ }^{4}$, Givanildo Roncatto ${ }^{1}$ \\ ${ }^{1}$ Embrapa Acre, Rodovia BR-364, Km 14, CP 321, CEP 69908-970, Rio Branco, AC, Brasil \\ ${ }^{2}$ Embrapa Florestas, Estrada da Ribeira, Km 111, CP 319, CEP 83411-000, Colombo, PR, Brasil \\ ${ }^{3}$ Embrapa Amazônia Oriental, Trav. Dr. Enéas Pinheiro, s/nº, CP 48, CEP 66095-100, Belém, PA, Brasil \\ ${ }^{4}$ Embrapa Algodão, Rua Osvaldo Cruz, 1.143, CP 174, CEP 58428-095, Campina Grande, PB, Brasil \\ ${ }^{5}$ Embrapa Meio Norte, Av. Duque de Caxias, 5.650, CEP 64066-220, Teresina, PI, Brasil
}

"Autor correspondente:

clideana@cpafac.embrapa.br

Termos para indexação:

Theobroma grandiflorum

Melhoramento

Seleção precoce

Index terms:

Theobroma grandiflorum

Breeding

Precocious election

Histórico do artigo:

Recebido em 08 mar 2010

Aprovado em 16 fev 2011

Publicado em 30 jun 2011

doi: 10.4336/2011.pfb.31.66.123
Resumo - O cupuaçuzeiro, espécie nativa da região Amazônica, encontra-se em processo de domesticação, apresentando ampla variabilidade genética e condições favoráveis para o estabelecimento de estratégias de melhoramento e conservação. $\mathrm{O}$ objetivo foi avaliar geneticamente 36 progênies de meios-irmãos de cupuaçu oriundos de seleção massal estratificada em áreas de produtores, com base nos componentes primários da produtividade. $\mathrm{O}$ experimento para seleção das progênies superiores foi instalado no delineamento aleatorizado em blocos, com quatro plantas por parcela e três repetições. Foram consideradas as variáveis de caracterização do fruto (comprimento e largura, ambos em centímetro) e componentes de produção avaliados em grama (peso médio de fruto por planta, peso médio de polpa com sementes por planta e peso médio de polpa por planta). Os dados foram analisados através da metodologia REML/BLUP com o emprego do software Selegen. Para realizar o agrupamento, utilizaram-se matrizes de distâncias euclidianas genéticas e de Mahalanobis. A população experimental possibilitou a seleção de parentais superiores para formação de população base para o melhoramento genético do cupuaçu. Das 36 progênies analisadas, apenas as progênies 9, 10 e 18 mostraram divergência genética, podendo ser recomendadas juntamente com alguma progênie do grupo 1 que mostrar superioridade agronômica para formar população base de clones experimentais com alta dissimilaridade genética.

\section{Genetic analysis of half-sib progenies of Theobroma grandiflorum}

\begin{abstract}
The cupuaçuzeiro (Theobroma grandiflorum), species native from the Amazon region, has been domesticated presenting a broad genetic variability, and thus favorable conditions for establishing of strategies to breeding and genetic conservation. The aim of this work was to evaluate 36 half-sib progenies of Theobroma grandiflorum obtained from a stratified mass selection in farmers areas based on primary components of yield. The experiment for selection of elite progenies was set up in a randomized complete block design with three replications and four plants per plot. The fruit traits (length and width, in $\mathrm{cm}$ ) and components of production (average weight of fruit/plant; average weight of pulp with seeds/plant and average weight of pulp/plant, in g) were evaluated. The data were analyzed through the methodology of REML/BLUP using the Selegen software. Analysis of grouping was performed using the matrix of genetic euclidean distances and Mahalanobis. The experimental population allowed the selection of superior parents for formation of base population to be used in the genetic improvement of cupuaçu. Among the 36 analyzed lineages only the genotypes 9,10 and 18 showed genetic divergence and they can be recommended with some lineage of group 1 that demonstrates agronomic superiority to form base population of experimental clones with high genetic dissimilarity.
\end{abstract}




\section{Introdução}

O cupuaçuzeiro, espécie nativa da região Amazônica, encontra-se em processo de domesticação, apresentando grande importância socioeconômica para a região (Moraes et al., 1994), ampla variabilidade genética e condições favoráveis para o estabelecimento de estratégias de melhoramento e conservação.

Os frutos do cupuaçuzeiro são muito apreciados pela indústria de alimentos, sendo por isso muito comercializados na região Norte do Brasil. A polpa apresenta sabor agradável e as sementes produzem uma gordura fina, da qual pode ser elaborado o cupulate, semelhante ao chocolate (Vasconcelos et al., 1975). Apesar de sua grande importância, a cultura apresenta baixa produtividade. Desse modo, programas de melhoramento são essenciais como forma de garantir a sustentabilidade de seu sistema produtivo (Alves \& Figueira, 2002).

Análises de distribuição da variabilidade genética, estimativas de parâmetros genéticos e predição de ganhos dependem dos diferentes ambientes em que se encontram os genótipos. Nas condições do Estado do Acre, onde a Embrapa está iniciando o programa de melhoramento genético do cupuaçuzeiro, este tipo de informação é escasso, porém imprescindível ao estabelecimento de estratégias de seleção e de conservação de germoplasma.

A predição de valores genéticos e a estimação de componentes de variância são atividades essenciais no melhoramento de plantas perenes, constituindo em técnicas ótimas de avaliação genética, sendo o REML/BLUP (máxima verossimilhança restrita/melhor predição linear não viciada) o procedimento padrão de estimação/predição é (Souza et al., 2002).

Em se tratando de espécie perene, o melhoramento do cupuaçu requer a realização de medidas repetidas em cada indivíduo, o que representa uma dificuldade intrínseca por envolver tempo geralmente elevado para recomendação de uma cultivar superior. A seleção precoce constitui, assim, uma alternativa mais ágil para a formação de populações-base.

O melhoramento apresenta resultados em consonância aos interesses dos produtores, resumindo-se em material produtivo ou elevada capacidade de frutificação, resistente a pragas e doenças e com adequadas características de adaptabilidade e estabilidade.

Este trabalho foi desenvolvido com o objetivo de identificar materiais genéticos promissores e promover seleção de progênies de cupuaçu, promover a clonagem dos melhores genitores e formar híbridos de polinização aberta entre as progênies com maior divergência genética.

\section{Material e métodos}

A população experimental do programa de melhoramento do cupuaçu da Embrapa Acre é constituída por 36 famílias de meios-irmãos obtidos a partir de seleção massal estratificada, em áreas de produtores, com base na produtividade e resistência a doenças.

O experimento constou de 36 progênies de meiosirmãos de cupuaçu, instaladas em delineamento aleatorizado em blocos, com quatro plantas por parcela e três repetições na área experimental da Embrapa Acre, Rio Branco, AC (0958'29'S, 6748'36'W; altitude de 153 m; clima Am, segundo a classificação de Köppen). As variáveis analisadas foram as de caracterização do fruto (comprimento e largura, ambos em $\mathrm{cm}$ ) e componentes de produção (peso médio, em grama, de fruto por planta; de polpa com sementes por planta e de polpa por planta).

Utilizou-se do seguinte modelo estatístico para a avaliação das 36 progênies no delineamento de blocos ao acaso com uma observação por parcela:

Modelo linear misto (modelo aditivo univariado)

$\mathrm{y}=\mathrm{Xb}+\mathrm{Za}+\mathrm{Wc}+\mathrm{e}$, em que

$\mathrm{y}, \mathrm{b}, \mathrm{a}, \mathrm{c}$ e e: vetores de dados, dos efeitos de blocos (fixos), dos efeitos genéticos aditivos (aleatórios), de efeitos de parcela (aleatórios) e dos erros aleatórios, respectivamente.

$\mathrm{X}, \mathrm{Z}$ e W: matrizes de incidência para $\mathrm{b}$, a e c, respectivamente.

$\mathrm{E}\left[\begin{array}{c}\mathrm{y} \\ \mathrm{a} \\ \mathrm{c} \\ \mathrm{e}\end{array}\right]=\left[\begin{array}{c}\mathrm{Xb} \\ 0 \\ 0 \\ 0\end{array}\right]$ e $\operatorname{Var}\left[\begin{array}{c}\mathrm{y} \\ \mathrm{a} \\ \mathrm{c} \\ \mathrm{e}\end{array}\right]=\left[\begin{array}{cccc}\mathrm{V} & \mathrm{ZG} & \mathrm{WC} & \mathrm{R} \\ \mathrm{GZ} & \mathrm{G} & 0 & 0 \\ \mathrm{CW}^{\prime} & 0 & \mathrm{C} & 0 \\ \mathrm{R} & 0 & 0 & \mathrm{R}\end{array}\right]$

em que:

$G=A \sigma_{a}^{2}$

$R=I \sigma_{c}^{2}$

$C=I \sigma_{e}^{2}$

$V=Z A \quad \sigma_{a}^{2} Z^{\prime}+W I \quad \sigma_{c}^{2} W^{\prime}+I \quad \sigma_{e}^{2}=Z G Z^{\prime}+W C W^{\prime}+R$. 


$$
h^{2}=\frac{\sigma_{\mathrm{a}}^{2}}{\sigma_{\mathrm{a}}^{2}+\sigma_{\mathrm{c}}^{2}+\sigma_{\mathrm{e}}^{2}}
$$

$\mathrm{h}^{2}$ : herdabilidade individual no sentido restrito no bloco;

$c^{2}=\sigma_{c}^{2}\left(\sigma_{a}^{2}+\sigma_{c}^{2}+\sigma_{e}^{2}\right)$ : correlação devida ao ambiente comum da parcela;

$\sigma_{\mathrm{a}}^{2}$ : variância genética aditiva;

$\sigma_{\mathrm{c}}^{2}$ : variância entre parcelas;

$\sigma_{\mathrm{e}}^{2}$ : variância residual (ambiental dentro de parcelas + não aditiva);

A : matriz de correlação genética aditiva entre os indivíduos em avaliação.

As soluções para as equações de modelo misto devem ser obtidas por métodos iterativos de resolução de sistemas de equações lineares, tais como o método de Gauss Seidel. A solução direta via inversão da matriz dos coeficientes é impossível na prática.

Visando à formação de híbridos de polinização aberta em população-base isolada, foi estimada a divergência genética entre progênies utilizando-se o agrupamento das progênies (pelo método de Tocher), por meio de matrizes de distâncias euclidianas genéticas e de Mahalanobis. Os coeficientes de correlação genética foram calculados pela correlação entre os Blup's univariados.

Todas as análises foram realizadas por meio de software Selegen-Reml/Blup (Resende, 2002).

\section{Resultados e discussão}

Nesse trabalho, os CVs experimentais das características peso médio do fruto, peso médio de polpa com sementes e peso médio de polpa foram muito altos, de $37,3 \%, 39,3 \%$ e $40,9 \%$ (Tabela 1), respectivamente. Os valores podem ser explicados tanto pelos altos coeficientes de variação genética de 27,42\%, 27,43\% e $26,89 \%$, para peso médio de frutos, peso médio de polpa com sementes e peso médio de polpa por planta, respectivamente (Tabela 1), como por perdas localizadas de plantas em certas repetições, contribuindo ao aumento do erro experimental. Alves \& Resende (2008) relatam coeficientes de variação genética $(\mathrm{CVg})$ variando de $27 \%$ a $88 \%$ no âmbito de progênie e de $38 \%$ a $123 \%$ no âmbito individual como estatísticas que revelam excelentes possibilidades para a seleção na população estudada. Os coeficientes de variação genética aditiva individual apresentaram altas magnitudes para as características físicas dos frutos com valores maiores que os respectivos coeficientes de variação ambiental (Tabela 1). Esse resultado indica condição favorável para seleção do material em estudo.

De acordo com Maia et al. (2009), o número de repetições é determinante para reduzir a variância fenotípica entre as médias das progênies. A raiz quadrada da herdabilidade ao nível de médias resulta nas acurácias seletivas (raiz quadrada da $\mathrm{h}^{2}$ ) na ordem de 0,8182 para comprimento dos frutos e 0,7707 para largura (Tabela 1), atestando boa precisão e controle das causas de variação ambiental de ordem aleatória, promovendo segurança na seleção.

É possível que a redução na taxa de sobrevivência dos indivíduos e a presença de plantas improdutivas, causas do desbalanceamento do experimento, tenham contribuído para as moderadas a baixas acurácias médias observadas; para as características: peso médio de frutos $(0,5375)$, peso médio de polpa com sementes $(0,5174)$ e peso médio de polpa $(0,4951)$. Para Resende (2001), plantas com altos valores genéticos preditos, mas com acurácia baixa, devem ser utilizadas com ressalvas.

Para os caracteres de componentes de variância avaliados, os valores intermediários de coeficientes de variação relativa $(0,37 ; 0,35$ e 0,33$)$ associados a um número razoável de repetições levaram a quocientes moderados de herdabilidade (confiabilidade) ao nível de médias de famílias $(0,29 ; 0,27$ e 0,25$)$, possibilitando acurácias medianas com valores situados próximos de $0,5(0,54 ; 0,52$ e 0,50$)$ da seleção de progênies. Neste contexto, são factíveis as boas expectativas de seleção para o incremento da produção nesta população base.

Ao se estimar a herdabilidade individual no sentido restrito $\left(\mathrm{h}^{2} \mathrm{a}=0,1625+/-0,145 ; 0,1611+/-0,145\right.$ e 0,1563 $+/-0,143)$, considera-se a dispersão genética aditiva: porção da variância genética atribuída a efeitos gênicos aditivos que são repassados para as progênies de meiosirmãos, por exemplo, via cruzamentos estocásticos. Neste caso, isso é pertinente pois trata-se de seleção populacional onde se explora a variância genética aditiva. Com respeito aos desvios apresentados, suas magnitudes não levam aquelas estimativas a assumirem o valor zero, o que é favorável e esperado para os carateres considerados. Estimativas de parâmetros genéticos em cupuaçuzeiro são raras. Souza et al. (2002) relatam estimativas de herdabilidade e repetibilidade para o caráter produção de frutos, da ordem de $25 \%$ 
e 30\%, respectivamente. Esses valores são superiores aos obtidos no presente trabalho e podem refletir as diferentes estruturas genéticas das populações usadas em cada trabalho.

Para Resende (2001), a seleção intrapopulacional é geralmente baseada em índice, incluindo as informações do indivíduo e da média da família, com recombinação dos próprios indivíduos avaliados. Isto conduz à maior intensidade de seleção e também à menor acurácia em relação à seleção de genitores.

A seleção com base em testes de progênies de meiosirmãos explora toda a variância genética aditiva na seleção realizada entre indivíduos na população como um todo, isto é, procedendo-se seleção entre e dentro das famílias. As estimativas dessa variância mostraramse significativas para todos os caracteres considerados. Adicionalmente, entre as características avaliadas, os caracteres relacionados ao rendimento se complementam e podem ser usados simultaneamente na escolha de parentais promissores para composição do lote de recombinação de primeiro ciclo, promovendo uma mistura equitativa dos alelos favoráveis dos materiais genéticos deixados para reprodução.

Uma seleção de parentais em uma população constituída por famílias de meios irmãos implica na existência de dispersão genética aditiva entre eles. A variância genética aditiva para as variáveis envolvidas na produtividade mostraram menor amplitude que suas respectivas variâncias de efeitos residuais (ambiental + não aditiva). A natureza quantitativa desses caracteres causa essa dispersão residual acentuada (Tabela 1).

As estimativas das variâncias dos efeitos ambientais apresentaram magnitudes relativamente baixas para as características menos influenciadas pelo ambiente como o são o comprimento e a largura do fruto com valores de 0,22 a 0,36 . Essas quantidades mostraram-se inferiores às variâncias genéticas aditivas $(3,74$ e 4,23) (Tabela 1$)$.

As famílias 10, 9, 26, 18, 3 e 12 se destacaram em produtividade de frutos por progênie ao ano. Em um grupo de baixo desempenho produtivo foram reunidas as progênies 24, 23, 33 e 34. Para o rendimento de polpa com sementes por progênie ao ano, as famílias 18, 10, 26,
8,12 e 3 mostraram superioridade. Apresentando menor peso médio de polpa com sementes por progênie ao ano, apareceram as famílias $34,23,24,33$ e 25 . O peso médio de polpa é uma característica altamente desejável quando o produto explorado é a polpa para processamento de derivados. As famílias que apresentaram elevados rendimentos de polpa foram 18, 10, 12, 29, 8 e 26 . As progênies com produção de polpa inferior foram 34,23 , 33 e 24 (Tabela 2).

Os maiores ganhos preditos simultaneamente para as características peso médio de fruto, peso médio de polpa com sementes e peso médio de polpa livre de sementes foram encontrados para as famílias 10, 26, 18 , e 12. Cabe ressaltar que não existe na literatura um parâmetro definido para comparar o desempenho do material experimental de cupuaçu. Uma seleção precoce e menos intensa pode ser orientada no sentido de compor a população de primeiro ciclo com vistas à realização de intercruzamentos em que haverá ampliação da variabilidade genética a ser explorada nos ciclos subsequentes (Tabela 2).

Os coeficientes de correlação genética entre as características peso médio de fruto e peso de polpa com sementes $(0,8860)$, peso do fruto e peso de polpa $(0,902)$ e peso de polpa e peso de polpa com sementes $(0,9702)$ apresentaram altas magnitudes. Este fato refletiu-se na classificação bastante conservada das famílias do grupo mais produtivo e do grupo de menor rendimento para características de produção.

Para Cruz (2005), uma das fases primordiais de um programa de melhoramento é a escolha de genitores a serem intercruzados para formar a população-base, na qual o pesquisador investirá seus esforços em busca de material genético de maior produtividade, qualidade e adaptação, sendo observados o desempenho relativo a vários atributos, a capacidade combinatória e a adaptação. Outro aspecto fundamental é a diversidade, esperando-se obter por meio de recombinações gênicas a complementaridade e a variabilidade indispensáveis para que a seleção seja praticada. 
Tabela 1. Estimativas de Componentes de Variância e parâmetros genéticos (REML Individual) para os variáveis de caracterização do fruto (comprimento do fruto - CF e largura do fruto - LF) e componentes de produção em g (peso médio (P) de fruto/planta; peso médio (P) de polpa com sementes/planta e peso médio (P) de polpa/planta.

\begin{tabular}{|c|c|c|c|c|c|c|}
\hline Estimativas & $\begin{array}{l}\mathrm{CF} \\
(\mathrm{cm})\end{array}$ & $\begin{array}{l}\mathbf{L F} \\
(\mathrm{cm})\end{array}$ & P (Fruto) & $\begin{array}{c}\mathbf{P} \\
(\text { polpa }+ \text { sem })\end{array}$ & $P$ (polpa) & Parâmetros \\
\hline $\mathrm{Va}$ & 3,7376 & 4,23057 & 21,2380 & 7,2133 & 3,8586 & Va: variância genética aditiva. \\
\hline Vparc & 0,2155 & 0,36629 & 10,4572 & 5,4038 & 3,9776 & Vparc: variância ambiental entre parcelas. \\
\hline Ve & 1,8704 & 4,03592 & 99,0274 & 32,1570 & 16,8475 & Ve: variância residual (ambiental + não aditiva). \\
\hline Vf & 5,8235 & 8,63278 & $* * * * *$ & 44,7742 & 24,6837 & $\mathrm{Vf}=\mathrm{Va}+\mathrm{Vparc}+\mathrm{Ve}:$ \\
\hline $\mathrm{h} 2 \mathrm{a}$ & $\begin{array}{l}0,6418 \\
+-0,289\end{array}$ & $\begin{array}{l}0,4901+- \\
0,253\end{array}$ & $\begin{array}{l}0,1625 \\
+-0,145\end{array}$ & $\begin{array}{l}0,1611 \\
+-0,145\end{array}$ & $\begin{array}{l}0,1563 \\
+-0,143\end{array}$ & $\begin{array}{l}\mathrm{h} 2 \mathrm{a}=\mathrm{Va} / \mathrm{Vf} \text { : herdabilidade individual no sentido } \\
\text { restrito, ou seja, dos efeitos aditivos. }\end{array}$ \\
\hline h2aj & 0,6665 & 0,5118 & 0,1766 & 0,1832 & 0,1864 & $\begin{array}{l}\text { h2aj }=\mathrm{Va} /(\mathrm{Va}+\mathrm{Ve}) \text { : herdabilidade individual no } \\
\text { sentido restrito, ajustada para os efeitos de } \\
\text { parcela. }\end{array}$ \\
\hline c2parc & 0,8182 & 0,0424 & 0,0710 & 0,1207 & 0,1611 & $\begin{array}{l}\text { c2parc = Vparc/Vf: coeficiente de determinação } \\
\text { dos efeitos de parcela. }\end{array}$ \\
\hline $\mathrm{h} 2 \mathrm{mp}$ & 0,6695 & 0,5940 & 0,2890 & 0,2677 & 0,2451 & $\begin{array}{l}\text { h2mp }=(0.25 \mathrm{Va}) /[0.25 \mathrm{Va}+\mathrm{Vparc} / 3+ \\
(0.75 \mathrm{Va}+\mathrm{Ve}) / 12]: \text { herdabilidade da média de } \\
\text { progênies, assumindo sobrevivência completa, } \\
\text { em que } 3 \text { é o número de repetições e } 12 \text { é o } \\
\text { número de repetições multiplicado pelo número } \\
\text { de plantas por parcela (4). }\end{array}$ \\
\hline Acprog & 0,8182 & 0,7707 & 0,5375 & 0,5174 & 0,4951 & $\begin{array}{l}\text { Acprog = raiz quadrada de h2mp: acurácia da } \\
\text { seleção de progênies, assumindo sobrevivência } \\
\text { completa. }\end{array}$ \\
\hline h2ad & 0,5998 & 0,4401 & 0,1386 & 0,1440 & 0,1466 & $\begin{array}{l}\mathrm{h} 2 \mathrm{ad}=(0.75 \mathrm{Va}) /(0.75 \mathrm{Va}+\mathrm{Ve}) \text { : herdabilidade } \\
\text { aditiva dentro de parcela. }\end{array}$ \\
\hline CVgi\% & 10,7419 & 6,6085 & 27,4229 & 27,4375 & 26,8878 & $\begin{array}{l}\mathrm{CVgi} \%=\left[(\mathrm{Va})^{1 / 2} / \text { Media Geral }\right] * 100: \\
\text { coeficiente de variação genética aditiva } \\
\text { individual. }\end{array}$ \\
\hline $\mathrm{CVgp} \%$ & 5,3710 & 3,3043 & 13,7115 & 13,7188 & 13,4439 & $\begin{array}{l}\mathrm{CVgp} \%:\left[(\mathrm{Va} / 4)^{1 / 2} / \mathrm{Media} \text { Geral }\right] * 100: \\
\text { coeficiente de variação genotípica entre } \\
\text { progênies. }\end{array}$ \\
\hline $\mathrm{Cve} \%$ & 6,5363 & 4,7314 & 37,2545 & 39,2955 & 40,86489 & $\begin{array}{l}\mathrm{CVe} \%=\left\{[(0.75 \mathrm{Va}+\mathrm{Ve}) / 3+\mathrm{Vparc}]^{1 / 2} /\right. \\
\text { Media Geral }\} * 100: \text { coeficiente de variação } \\
\text { experimental. }\end{array}$ \\
\hline $\mathrm{CVr}$ & 0,8217 & 0,6984 & 0,3680 & 0,34912 & 0,3290 & $\begin{array}{l}\mathrm{CVr}=\mathrm{CVgp} / \mathrm{CVe}=\text { coeficiente de variação } \\
\text { relativa. }\end{array}$ \\
\hline PEV & 0,3088 & 4,2938 & 3,7753 & 1,3205 & 0,7282 & $\begin{array}{l}\mathrm{PEV}=\left(1-\mathrm{Acprog}^{2}\right) \mathrm{Va} / 4 \text { : variância do erro de } \\
\text { predição dos valores genotípicos de progênie, } \\
\text { assumindo sobrevivência completa. }\end{array}$ \\
\hline SEP & 0,5557 & 2,0721 & 1,9430 & 1,1491 & 0,8534 & $\begin{array}{l}\mathrm{SEP}=\text { raiz quadrada da } \mathrm{PEV} \text { : desvio padrão do } \\
\text { valor genotípico predito de progênie, assumindo } \\
\text { sobrevivência completa. }\end{array}$ \\
\hline Média & 17,9976 & 9,8422 & 16,8052 & 9,7887 & 7,3057 & Média geral do experimento. \\
\hline
\end{tabular}


Tabela 2. Predição dos Componentes de Média (Blup Individual) para os variáveis de componentes de produção em $\mathrm{g}$ (peso médio (P) de fruto/planta; peso médio (P) de polpa com sementes/planta e peso médio (P) de polpa/planta.

\begin{tabular}{|c|c|c|c|c|c|c|c|c|}
\hline \multicolumn{9}{|c|}{ Seleção de genitores } \\
\hline \multicolumn{3}{|c|}{$P$ (fruto) } & \multicolumn{3}{|c|}{ P (polpa+sems) } & \multicolumn{3}{|c|}{$\mathbf{P}$ (polpa) } \\
\hline Genitor & Ganho & Nova média & Genitor & Ganho & Nova média & Genitor & Ganho & Nova média \\
\hline 10 & 5,3313 & 22,1365 & 18 & 3,7068 & 13,4954 & 18 & 2,4050 & 9,7103 \\
\hline 9 & 4,9383 & 21,7438 & 10 & 3,3183 & 13,1070 & 10 & 2,0815 & 9,3872 \\
\hline 26 & 4,3303 & 21,1355 & 26 & 2,6651 & 12,4538 & 12 & 1,7641 & 9,0698 \\
\hline 18 & 3,9567 & 20,7619 & 8 & 2,2649 & 12,0535 & 29 & 1,5811 & 8,8868 \\
\hline 3 & 3,5669 & 20,3721 & 12 & 2,0193 & 11,8083 & 8 & 1,4477 & 8,7534 \\
\hline 12 & 3,2618 & 20,0670 & 3 & 1,8371 & 11,6258 & 26 & 1,3526 & 8,6583 \\
\hline 20 & 3,0399 & 19,8451 & 29 & 1,7025 & 11,4912 & 2 & 1,2568 & 8,5624 \\
\hline 13 & 2,8670 & 19,6722 & 20 & 1,5978 & 11,3864 & 3 & 1,1671 & 8,4728 \\
\hline 2 & 2,7258 & 19,5310 & 22 & 1,5028 & 11,2914 & 22 & 1,0925 & 8,3982 \\
\hline 8 & 2,6021 & 19,4073 & 2 & 1,4250 & 11,2136 & 9 & 1,0294 & 8,3351 \\
\hline 29 & 2,4857 & 19,2909 & 3 & 1,3510 & 11,1397 & 13 & 0,9734 & 8,2790 \\
\hline 22 & 2,3422 & 19,1474 & 9 & 1,2750 & 11,0636 & 17 & 0,9125 & 8,2181 \\
\hline 28 & 2,2110 & 19,0162 & 28 & 1,1986 & 10,9872 & 20 & 0,8593 & 8,1646 \\
\hline 35 & 2,0779 & 18,8831 & 36 & 1,1076 & 10,8962 & 21 & 0,8054 & 8,1111 \\
\hline 36 & 1,9559 & 18,7610 & 21 & 1,0280 & 10,8166 & 36 & 0,7572 & 8,0629 \\
\hline 30 & 1,8435 & 18,6487 & 35 & 0,9517 & 10,7403 & 28 & 0,7125 & 8,0181 \\
\hline 7 & 1,7185 & 18,5237 & 11 & 0,8812 & 10,6699 & 30 & 0,6661 & 7,9718 \\
\hline 19 & 1,5970 & 18,4022 & 7 & 0,8163 & 10,6050 & 7 & 0,6224 & 7,9281 \\
\hline 21 & 1,4869 & 18,2920 & 19 & 0,7576 & 10,5463 & 11 & 0,5811 & 7,8867 \\
\hline 16 & 1,3783 & 18,1835 & 4 & 0,7034 & 10,4920 & 32 & 0,5436 & 7,8492 \\
\hline 17 & 1,2705 & 18,0757 & 16 & 0,6542 & 10,4429 & 35 & 0,5087 & 7,8144 \\
\hline 15 & 1,1681 & 17,9733 & 17 & 0,6081 & 10,3968 & 19 & 0,4698 & 7,7755 \\
\hline 32 & 1,0739 & 17,8791 & 30 & 0,5660 & 10,3546 & 4 & 0,4308 & 7,7365 \\
\hline 4 & 0,9873 & 17,7925 & 15 & 0,5267 & 10,3154 & 14 & 0,3936 & 7,6993 \\
\hline 1 & 0,9017 & 17,7069 & 32 & 0,4890 & 10,2777 & 5 & 0,3586 & 7,6643 \\
\hline 11 & 0,8207 & 17,6259 & 1 & 0,4480 & 10,2367 & 16 & 0,3251 & 7,6308 \\
\hline 14 & 0,7451 & 17,5503 & 5 & 0,4078 & 10,1964 & 15 & 0,2937 & 7,5993 \\
\hline 5 & 0,6711 & 17,4763 & 27 & 0,3661 & 10,1548 & 27 & 0,2638 & 7,5695 \\
\hline 31 & 0,6004 & 17,4056 & 31 & 0,3237 & 10,1124 & 1 & 0,2333 & 7,5390 \\
\hline 27 & 0,5324 & 17,3376 & 14 & 0,2828 & 10,0714 & 31 & 0,2048 & 7,5105 \\
\hline 6 & 0,4475 & 17,2527 & 6 & 0,2374 & 10,0260 & 6 & 0,1724 & 7,4781 \\
\hline 25 & 0,3575 & 17,1626 & 25 & 0,1936 & 9,9823 & 25 & 0,1385 & 7,4442 \\
\hline 34 & 0,2718 & 17,0769 & 33 & 0,1442 & 9,9328 & 24 & 0,1020 & 7,4077 \\
\hline 33 & 0,1824 & 16,9876 & 24 & 0.0947 & 9,8833 & 33 & 0,0671 & 7,3728 \\
\hline 23 & 0,0971 & 16,9023 & 23 & 0,4642 & 9,8350 & 23 & 0,0342 & 7,3399 \\
\hline 24 & 0,0000 & 16,8052 & 34 & 0,0000 & 9,7887 & 34 & 0,0000 & 7,3057 \\
\hline
\end{tabular}


Espécies que apresentam oportunidade para multiplicação vegetativa representam um fator facilitador para o melhoramento, aumentando significativamente as possibilidades de ganho em eficiência do processo seletivo. Ao considerar a seleção individual de plantas superiores e sua clonagem, está-se explorando toda variância genética e reproduzindo integralmente seu genoma. As plantas: 9.3, 10.4, 10.2, 18.2, 18.4 e 10.1 apresentaram melhor classificação quanto ao peso médio do fruto, da polpa e polpa com sementes (Tabela 3). Técnicas de propagação vegetativa por estaquia e propagação in vitro encontram-se em desenvolvimento no cupuaçuzeiro, visando acelerar a produção em larga escala de clones selecionados nos programas de melhoramento (Ferreira et al., 2005; Ledo et al., 2002).

Pelo agrupamento (Tocher) de distâncias euclidianas quadradas genéticas, determinaram-se os dois grupos de progênies. Grupo 1: 1, 2, 3, 4, 5, 6, 7, 8, 9, 11, 12, $13,14,15,16,17,19,20,21,22,23,24,25,26,27$, $28,29,30,33,34,35,36,31,32$, e um segundo grupo reuniu as progênies 10 e 18 . Através do agrupamento (Tocher) de distâncias genéticas Mahalanobis quadradas genéticas, foram disjuntos três grupos. No grupo 1 , as seguintes progênies foram classificadas por similaridade: $1,2,3,4,5,6,7,8,10,11,12,13,14$, $15,16,17,19,20,21,22,23,24,25,26,27,28,29$, $30,31,32,33,34,35,36$; o grupo 2 ficou composto pela progênie 9 e um terceiro grupo ficou formado pela progênie 18. Houve alta concordância entre as duas medidas de similaridade. Das 36 progênies analisadas, apenas as progênies 9, 10 e 18 mostraram divergência genética, podendo ser recomendadas juntamente com alguma progênie do grupo 1 que mostrar superioridade agronômica para implantação de experimentos com clones experimentais com alta dissimilaridade genética, condição desejável, já que o cupuaçu é espécie que apresenta autoincompatibilidade, afim de serem testados em vários ambientes representativos das condições onde aqueles materiais serão recomendados.

A alta concentração de progênies reunidas no primeiro grupo pelas duas metodologias permitiu inferir que as famílias que compõem a população de trabalho do programa de melhoramento apresentam mesma procedência.

Tabela 3. Predição dos Componentes de Média (Blup Individual) para os variáveis de componentes de produção em g (peso médio $(\mathrm{P})$ de fruto/planta; peso médio $(\mathrm{P})$ de polpa com sementes/planta e peso médio (P) de polpa/planta.

\begin{tabular}{ccccccccc}
\hline \multicolumn{7}{c}{ P (fruto) } & \multicolumn{3}{c}{ Peleção de indivíduos } \\
\hline Planta $^{1}$ & Ganho & Nova média & Planta ${ }^{1}$ & Ganho & Nova média & Planta $^{(1)}$ & Ganho & Nova média \\
\hline $9.3 \mathrm{~B} 1$ & 12.4316 & 29.2367 & $18.2 \mathrm{~B} 1$ & 7.5579 & 17.3465 & $18.2 \mathrm{~B} 1$ & 5.1778 & 12.4835 \\
$10.4 \mathrm{~B} 2$ & 9.3926 & 26.1978 & $10.4 \mathrm{~B} 2$ & 5.6857 & 15.4744 & $10.4 \mathrm{~B} 2$ & 3.8557 & 11.1614 \\
$10.2 \mathrm{~B} 3$ & 8.2180 & 25.0232 & $10.2 \mathrm{~B} 3$ & 4.8311 & 14.6198 & $12.4 \mathrm{~B} 1$ & 3.2992 & 10.6049 \\
$18.2 \mathrm{~B} 1$ & 7.5574 & 24.3626 & $18.4 \mathrm{~B} 1$ & 4.3904 & 14.1791 & $10.1 \mathrm{~B} 1$ & 3.0187 & 10.3244 \\
$10.2 \mathrm{~B} 1$ & 6.9760 & 23.7812 & $10.1 \mathrm{~B} 2$ & 4.0985 & 13.8871 & $29.2 \mathrm{~B} 2$ & 2.8007 & 10.1064 \\
$10.1 \mathrm{~B} 2$ & 6.5646 & 23.3698 & $10.1 \mathrm{~B} 1$ & 3.8827 & 13.6714 & $18.4 \mathrm{~B} 1$ & 2.6353 & 9.9410 \\
\hline
\end{tabular}

(1) indivíduos selecionados da população experimental.

Uma população em equilíbrio de espécies alógamas ou panmíticas é constituída prevalecentemente por uma mistura de híbridos onde se estabelece uma complementação acentuada ao nível de genoma entre os indivíduos que se intercruzam. $\mathrm{O}$ vigor de híbrido devido à heterose deve ser buscado e mantido em uma população de cruzamentos melhorada. Neste contexto, espera-se que a seleção de parentais alocados em grupos divergentes geneticamente redunde em formação de população com complementação gênica apreciável. A seleção incluiu as famílias 9; 10 e 18 dos grupos menores e as progênies $3 ; 8 ; 12 ; 26$ e 29 do grupo 1.
É esperado que haja otimização no esquema seletivo precoce, que visa à implementação de experimentos com materiais produtivos e complementares na composição da população de primeiro ciclo, para os indivíduos que se sobressaíram e que foram selecionados para os componentes primários da produção, tendo também suas famílias $(9,10,1812)$ alocadas em grupos divergentes.

\section{Conclusões}

- Há indivíduos superiores para formação de população-base para o melhoramento genético do cupuaçu na população experimental. 
- Apenas as progênies 9; 10 e 18 mostram divergência genética e são recomendadas, juntamente com algumas progênies do grupo 1 que mostraram superioridade agronômica, para formar a população de primeiro ciclo do programa de melhoramento.

\section{Agradecimentos}

À Funtac/CNPq, Fundação de Tecnologia do Estado do Acre/Conselho Nacional de Desenvolvimento Científico e Tecnológico, instituições financiadoras do projeto, e à Embrapa Acre, pelo apoio na condução do experimento.

\section{Referências}

ALVES, R.M.; FIGUEIRA, A. Cupuassu (Theobroma grandiflorum) genetic resources and breeding in the Brazilian Amazon. Ingenic Newsletter, v.7, p.25-32, 2002.

ALVES, R.; RESENDE, M. D. V. de. Avaliação genética de indivíduos e progênies de cupuaçuzeiro no Estado do Pará e estimativas de parâmetros genéticos. Revista Brasileira de Fruticultura, Jaboticabal v. 30, n. 3, p. 696-701, 2008.

CRUZ, C. D. Princípios de genética quantitativa. Viçosa. Editora UFV. 2005. 394 p.

FERREIRA, M. G. R.; CARVALHO, C.H.S; CARNEIRO, A.A. Indução de embriogênese somática em cupuaçu. Revista Brasileira de Fruticultura, Jaboticabal, v. 27, n.3, p. 500-503, 2005.
LEDO, A.S.; LAMEIRA, O.A.; BENBADIS,A.K. Explantes de cupuaçuzeiro submetidos a diferentes condições de cultura in vitro. Revista Brasileira de Fruticultura, Jaboticabal, v. 24, n.3, p. 604-607, 2002.

MAIA, M. C. C.; RESENDE, M. D. V. de; PAIVA, J. R. de; CAVALCANTE, J. J. V.; BARROS, L. M. de. Seleção simultânea para produção, adaptabilidade e estabilidade genotípicas em clones de cajueiro, via modelos mistos. Revista de Pesquisa Agropecuária Tropical, Goiânia, v. 39, n.1, p. 43-50, 2009.

MORAES, V. H. F; MULLER, C.H.; SOUZA, A. C. Native fruit species of economic potential from the Brazilian Amazom. Angewandte Botanik, v. 68, p.47-52, 1994

RESENDE, M. D. V. de. Melhoramento de Espécies Perenes. In: NASS, L. L.; VALOIS, A. C. C.; MELO, I. S. de; VALADARESINGLIS, M. C. (Eds.). Recursos genéticos em melhoramento de plantas. Rondonópolis: Fundação MT, 2001. p. 357-422.

RESENDE, M. D. V. de. Software Selegen-REML/BLUP. Colombo: Embrapa Florestas, 2002. 67 p. (Embrapa Florestas. Documentos, 77).

SOUZA, A. G. C.; RESENDE, M.D.V.; SILVA, S.E.L.; SOUZA, N. R. The cupuaçu genetic improvement program at. Embrapa Amazônia Ocidental. Crop Breeding and Applied Biotechnology, n. 2, p. 471-478, 2002.

VASCONCELOS, M. N. L.; SILVA, M. L.; MAIA, J. G. S. S; GOTTLIEB, O. R. Estudo químico das sementes do cupuaçu. Acta Amazônica, v. 5, n. 3, p. 293-295, 1975. 